\title{
ESTUDIO MINEROLÓGICO DE SUELOS AGRÍCOLAS POR ESPECTROSCOPIA MÖSSBAUER
}

\author{
María L.. Cerón L., Julio Fabián S. y Jorge A. Bravo C.
}

SUMILLA: Los compuestos de hierro (Fe) son muy comunes en la naturaleza. En el caso de suelos, se presenta en forma de sesquióxidos de hierro, tales como óxidos, hidróxidos y oxihidróxidos, así como cationes de sustitución en arcillas. Estos minerales se presentan en estado cristalino y amorfo. Por ser isotópicamente selectiva, la espectroscopía Mössbauer (resonancia gamma nuclear) permite identificar los minerales que contienen Fe aún cuando éstos están presentes en muy bajas concentraciones y en estado amorfo. En este trabajo reportamos los resultados preliminares del estudio que se viene realizando de muestras de suelos colectadas de la zona de Chinchero, Cusco. Los datos experimentales se obtuvieron por espectroscopía Mössbauer y difracción de rayos $x$; estos datos se complementaron con datos reportados por otros investigadores. En la preparación de las muestras se utilizó procedimientos establecidos y los espectros Mössbauer se tomaron a temperaturas de ambiente y nitrógeno líquido.

ABSTRACT: Iron (Fe) compounds are very common in nature. In the case of soils, it occurs in the form of Fe sesquioxides, such as oxides, hidroxides and oxyhydroxides, as well as substitution cations in clay minerals. These minerals occur in crystalline and amorphous states. The isotopic selective nature of Mössbauer spectroscopy (nuclear gamma resonance) allows the identification of minerals containing Fe even when they are present in low concentrations and in the amporphous state. In this work we report the preliminary results of a current study of soil samples collected from the area of Chinchero, Cusco. The experimental data were obtained by Mössbauer spectroscopy and X-ray diffraction; these data were complemented with data reported by other workers. Standard procedures were used for sample preparation and the Mössbauer spectra were taken at room and liquid nitrogen temperatures.

\section{INTRODUCCIÓN}

Este trabajo trata del estudio de la mineralogía de suelos agrícolas por la técnica de espectroscopia Mössbauer, EM, utilizando la transición resonante nuclear de g14,4keV del isótopo natural Fe-57, la cual nos permite detectar y medir en forma selectiva la presencia de sesquióxidos y otros minerales que contienen hierro (Fe), tales como los óxidos, hidróxidos, oxihidróxidos de hierro y las arcillas, que están presentes en los suelos. No existen estudios en nuestro país anteriores aplicando esta técnica para este fin. La Figura 1. muestra la ubicación de las unidades litoestratigráficas(1) de donde se recolectaron las muestras estudiadas. Los sitios M1, M2 y M3 corresponden a la formación Chinchero y se ubican al lado norte del pueblo de Chinchero, a 20km al Norte de la ciudad del Cusco, Perú. Los sitios M4 y M5 están ubicados al lado este de la pampa de Huaypo, frente al pueblo de Chinchero; corresponden a depósitos fluviales. De cada sitio se extrajo muestras de diferentes profundidades: superficie, 0,50m y 1,0m.

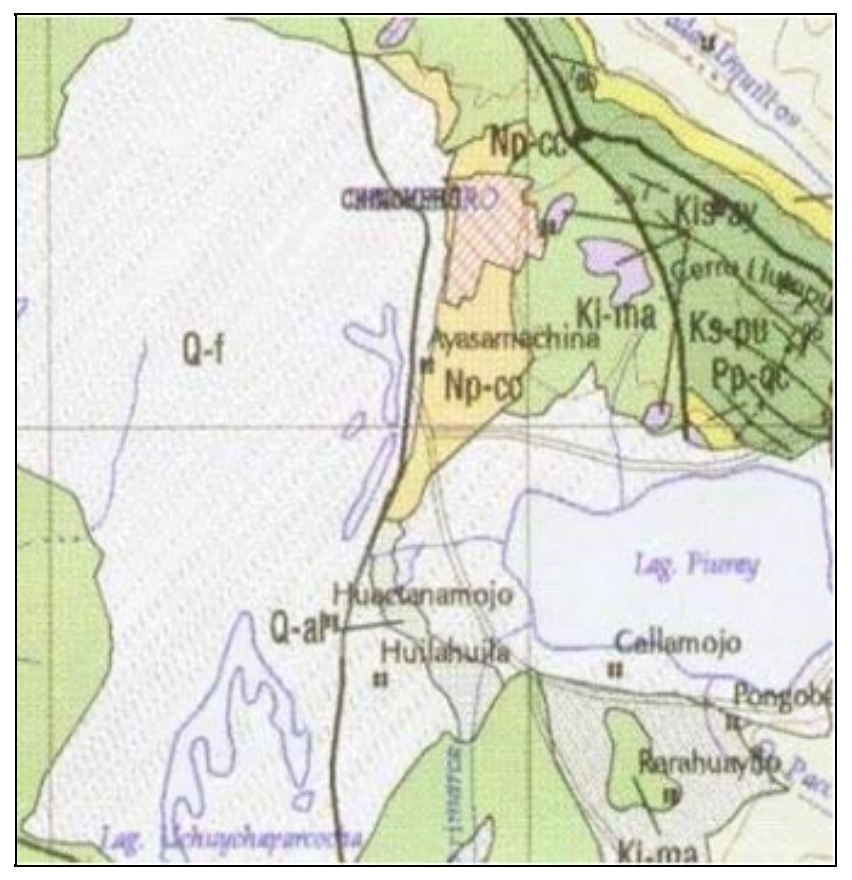

Figura 1. Mapa Geológico de la localidad de Chinchero, Cusco y alrededores

En este trabajo se reporta el avance en los resultados obtenidos en el análisis de las muestras de cuatro sitios denominándolos: M2 (suelo cultivado), M3 (suelo no cultivado), M4 (suelo cultivado) y M5 (suelo cultivado). Todas las muestras, motivo de este reporte, provienen de la superficie y $50 \mathrm{~cm}$ de profundidad.

Además de EM también se utiliza el análisis por difracción de rayos x, DRx, para obtener información cualitativa sobre la composición mineralógica de las muestras, en particular de los minerales que no contienen hierro. La identificación de cada mineral 
se fundamenta en la información inicial que proporciona la DRx y en la información sobre los parámetros hiperfinos de los minerales de Fe que proporciona la EM. Merece mencionar que los difractogramas de rayos x muestran limitaciones debido a la superposición de las reflexiones de los distintos compuestos de Fe entre sí y con otras especies cristalinas presentes en el suelo, en particular cuarzo y feldespato.

\section{PARTE EXPERIMENTAL}

Para el análisis instrumental de las muestras se procedió de la siguiente manera:

a)Preparación de las muestras: Las muestras fueron secadas a temperatura de ambiente y luego tamizadas en malla de $2 \mathrm{~mm}$. Para los análisis por DRx se utilizaron muestras sin tratamiento químico. Para los análisis por EM las muestras fueron adicionalmente pasadas por un tamiz de $45 \mathrm{~mm}$ y molidas en un mortero antes de colocarlas en el portamuestras; inicialmente se utilizó 160mg y posteriormente 300mg para una mejor optimización del arreglo experimental.

b)Tratamiento químico: Estas muestras fueron tratadas por el método DCB (Ditionito Citrato Bicarbonato) de Mehra-Jackson. Este método permite la eliminación de óxidos de Fe con estructuras de buena cristalización.

c) Análisis por DRx: Luego se procedió a las mediciones por DRx y efecto Mössbauer de todas estas muestras, tratadas y sin tratar. Para las mediciones por DRx se solicitó la colaboración del Laboratorio de DRx del Centro Brasileiro de Pesquisas Físicas, Brasil, el cual cuenta un difractómetro modelo TuR M62. Los difractogramas se tomaron con radiación Ka de Cu, con una resolución de $0.02^{\circ}$ paso de $2 \mathrm{~s}$, y cubren el rango de $10^{\circ}<2 \mathrm{q}<35^{\circ}$. No se prepararon muestras orientadas para estos fines.

d) Análisis por EM: Para las mediciones por EM se utilizó un arreglo de transmisión y fueron hechas a temperatura de ambiente, TA, y a temperatura de nitrógeno líquido (82K), TNL, Se utilizó una fuente de Co-57 en matriz de rhodio con una actividad promedio de $9 \mathrm{mCi}$ aproximadamente durante el periodo de este trabajo experimental. Para almacenar los espectros se utilizó 512 y 1024 canales. EM tiene ventajas sobre DRx en todo lo que respecta a la identificación de minerales que contienen hierro por ser EM una técnica isotópicamente selectiva. Para los ajustes de los espectros se utilizó el programa NORMOS, para sitios y distribuciones.

e) Medidas a TNL: Con el propósito de discernir la presencia de pequeñas partículas amorfas de goetita, a través de efectos superparamagnéticos, se tomó espectros de las muestras M2 y M4 a TNL.

\begin{tabular}{|c|c|c|c|c|}
\hline \multicolumn{5}{|c|}{ Leyenda de la Localidad de Chinchero: } \\
\hline Eraterma & Sistema & Serie & Unidades Litoestratigráficas & Sitios \\
\hline \multirow{2}{*}{ C E N O Z O I C A } & CU AT E R N A R I O & HOL OCENO & Depósitos fluviales Q-F & M4 - M5 \\
\hline & NEOGE N O & PLIOCENO & Fam. Chinchero Np - cc & $\mathrm{M} 2-\mathrm{M} 3$ \\
\hline
\end{tabular}

\section{RESULTADOS}

Las figuras 2a) a 2h) muestran los espectros Mössbauer obtenidos y analizados. Las figuras 3a) y 3b) muestran dos difractogramas característicos obtenidos.

Figura 2. Espectros Mössbauer de las Muestras de Suelos.

a) Muestra M2, sin tratar, a TA

b. Muestra M2, sin tratar, a TNL

c) Muestra M2, tratada con DCB, a TA

d) Muestra M4, tratada con DCB, a TA

e) Muestra M4, sin tratar, a TA

f) Muestra M4, sin tratar, a $80 \mathrm{~K}$

g) Muestra M3, sin tratar, a TA

h) Muestra M5, sin tratar, TA

Por EM se ha identificado los siguientes minerales:

i) Hematita (a - Fe2O3)

ii) Magnetita (a - Fe3O4)

iii)Illita (KxAl2(Si4-xAlx)O10(OH)2),0,5<x<0,75

iv)Muscovita (KAl2(AlSi3)O10(OH)2)

v) Montmorillonita (Al-Mg-Si-O-OH-Na)

vi) Lepidocrocita (g-FeOOH)

vii)Goetita (a-FeOOH)

viii)Ferrihidrita (Fe5O7(OH).4H2O)

Para los parámetros hiperfinos que se listan en las tablas se ha tomado en cuenta lo siguiente: el corrimiento isomérico, ISO, se da en $\mathrm{mm} / \mathrm{s}$ con respecto a una matriz de rhodio; el campo magnético hiperfino, Hm, se da en teslas, $\mathrm{T}$, y el corrimiento cuadrupolar, 2e, o desdoblamiento cuadrupolar, QUA, según sea el caso, se da en mm/s.

La tabla I lista los parámetros hiperfinos de las muestras sin tratar, superficie $(<2 \mathrm{~mm}$ y $<45 \mu \mathrm{m})$ y $50 \mathrm{~cm}(<2 \mathrm{~mm})$, correspondientes a los cuatro sitios, medidos a temperatura de ambiente, TA. La tabla II lista los parámetros hiperfinos de los espectros tomados a 
TNL para muestras de la superficie $(<2 \mathrm{~mm})$. La tabla III lista los parámetros hiperfinos respectivos para las muestras de la superficie $(<2 \mathrm{~mm})$ tratadas con el método "DCB" a TA.

Las tablas IV y V listan las áreas de absorción resonante, es decir, los valores de la absorción nuclear resonante integrada respectivas, de las mismas muestras cuyos parámetros hiperfinos aparecen en la tabla I. La tabla VI lista las áreas de absorción correspondientes a la tabla II. La tabla VII lista estas áreas correspondientes a la tabla III. Estas áreas se expresan en mm/s luego de normalizar el continuo a la unidad.

Se observa que illita, una arcilla, es el único mineral que se encuentra presente en todas las muestras. Hematita resulta ser el óxido de mayor concentración en las muestras M2 y M3, lo cual se manifiesta en la coloración rojiza de estas muestras. Es interesante notar que magnetita se encuentra sólo en la muestra M3 y en proporciones pequeñas apenas perceptibles. Por otro lado, sólo en la muestra M4 se ha encontrado goetita y ferrihidrita.

Al comparar los espectros de las muestras tratadas con DCB con los de las muestras sin tratamiento químico, se observa una menor intensidad en los picos que corresponden a óxidos como hematita, que se presentan con buena cristalización, que desaparecen por acción del tratamiento químico.

En el espectro de la muestra M2- superficie tomada a TNL no se observa la presencia de goetita magnética, lo cual descarta la presencia de goetita paramagnética en el espectro a TA. Asimismo, se observa un ligero aumento en el campo magnético hiperfino de la hematita con respecto a su valor a TA.

A TNL en la muestra M4 se puede observar

la aparición del sexteto magnético de la goetita, lo cual es una manifestación de superparamagnetismo en este mineral cuando éste se presenta en forma de pequeñas partículas. Este efecto no se observa en la muestra M2. Este resultado fue muy útil para dilucidar la presencia de goetita paramagnética en el espectro a TA.

La muestra M5-superficie- exhibe la menor concentración de Fe en todas las muestras aquí reportadas; muestra sólo dos desdoblamientos cuadrupolares adjudicados a la illita y montmorillonita.

En la tabla VIII se lista las distancias interplanares y las intensidades relativas de los picos característicos de difracción de rayos x de los minerales de hierro y otros de ocurrencia frecuente en las muestras estudiadas.

En todos los difractogramas de rayos-x se observa la presencia del pico intenso de cuarzo de 3.35A. Sólo en la muestra M5 se observa la presencia de calcita. Adicionalmente muestran la presencia de diferentes alúmino- silicatos. Se observa el solapamiento de picos de cuarzo y montmorillonita con los de illita, así como los picos de hematita solapados con los de goetita (Figuras 3a y 3b.). Así mismo, también se observa solapamientos de picos pequeños que hacen muy difícil definir con claridad a que especies minerales pertenecen.

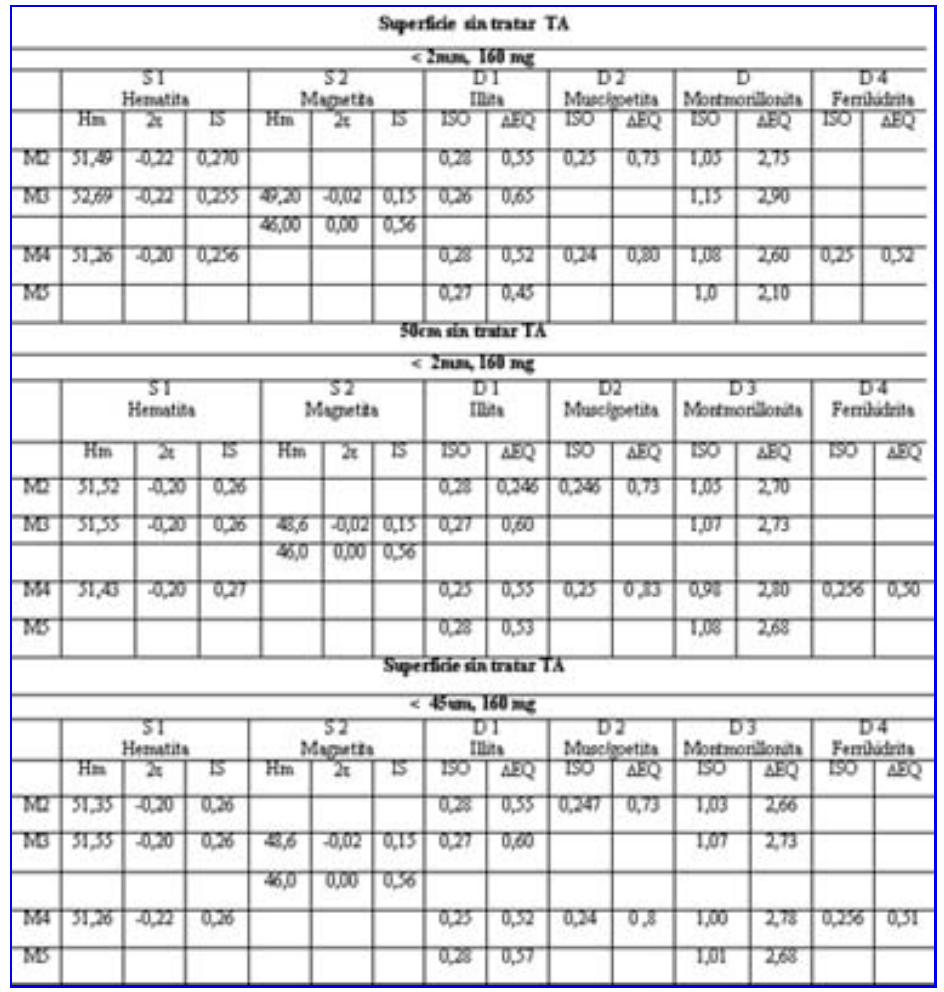


TABLA I. Parámetros hiperfinos de las especies minerales identificadas en muestras de suelos sin tratar

\begin{tabular}{|c|c|c|c|c|c|c|c|c|c|c|c|c|c|c|}
\hline \multicolumn{15}{|c|}{ Squetich tin trates, TIL } \\
\hline \multicolumn{15}{|c|}{$<2 \mathrm{~mm}, 160 \mathrm{mg}$} \\
\hline & \multicolumn{3}{|c|}{$\begin{array}{c}S 1 \\
\text { Hematita }\end{array}$} & \multicolumn{3}{|c|}{$\begin{array}{c}32 \\
\text { Goetts }\end{array}$} & \multicolumn{2}{|c|}{$\begin{array}{c}\text { D1 } \\
\text { Mosoonts }\end{array}$} & \multicolumn{2}{|c|}{$\begin{array}{l}\text { D2 } \\
\text { Dhte }\end{array}$} & \multicolumn{2}{|c|}{$\begin{array}{c}\text { D3 } \\
\text { Femistint }\end{array}$} & \multicolumn{2}{|c|}{$\begin{array}{c}\text { D4 } \\
\text { Montmonilio } \\
\end{array}$} \\
\hline & $\mathrm{Hm}$ & $2 x$ & is & $\mathrm{Hm}$ & $2 \pi$ & is & 150 & $\triangle D Q$ & 190 & $\triangle B Q$ & 180 & $\triangle D Q$ & 150 & $\triangle D Q$ \\
\hline $\mathrm{MD}$ & 53,14 & $-0,197$ & 0,270 & & & & 0,738 & 0,36 & 0,20 & 0,00 & & & 1,06 & 29 \\
\hline MA & 53,0 & 0,120 & 0,330 & 40,0 & $-0,28$ & 0,36 & & & 0,23 & 0,93 & 0,33 & 0,89 & 0,98 & 298 \\
\hline & & & & & & & & & 1,30 & 2,43 & & & & \\
\hline
\end{tabular}

TABLA II. Parámetros hiperfinos de las minerales identificados en muestras de suelos sin tratar

\begin{tabular}{|c|c|c|c|c|c|c|c|c|c|c|c|}
\hline \multicolumn{12}{|c|}{ Superficie, tratads cosDCB, TA. } \\
\hline \multicolumn{12}{|c|}{$<2 \mathrm{~mm}, 300 \mathrm{ng}$} \\
\hline & \multicolumn{3}{|c|}{$\begin{array}{c}\text { S1 } \\
\text { Hematita }\end{array}$} & \multicolumn{2}{|c|}{$\begin{array}{c}\text { D } 1 \\
\text { inita }\end{array}$} & \multicolumn{2}{|c|}{$\begin{array}{c}\text { D2 } \\
\text { Muscowíostita }\end{array}$} & \multicolumn{2}{|c|}{$\begin{array}{c}\text { D3 } \\
\text { Mottmonillo }\end{array}$} & \multicolumn{2}{|c|}{$\begin{array}{c}\text { D4 } \\
\text { Femhidinta }\end{array}$} \\
\hline & $\mathrm{Hm}$ & 150 & $\triangle \mathrm{EQQ}$ & 150 & $\triangle B Q$ & tSO & $\triangle E Q$ & 150 & $\triangle \mathrm{EQ}$ & & \\
\hline$M 2$ & 51,48 & 0,27 & $-0,22$ & 028 & 0,525 & 0,25 & 0,73 & 1,057 & 2,75 & & \\
\hline M4 & 51,24 & 0,246 & $.0,20$ & 0,28 & 0,550 & 0,25 & 0,80 & 1,04 & 2,69 & 0,35 & 0,50 \\
\hline
\end{tabular}

TABLA III. Parámetros hiperfinos de las muestras de minerales identificadas en muestras de suelos tratadas

\begin{tabular}{|c|c|c|c|c|c|c|c|c|}
\hline \multicolumn{9}{|c|}{$<2 \mathrm{~mm}$ steperficie sin tratar, TA } \\
\hline & \multicolumn{2}{|c|}{ MUESTRA M2 } & \multicolumn{2}{|c|}{ MUESTRA MB } & \multicolumn{2}{|c|}{ MUESTRA M4 } & \multicolumn{2}{|c|}{ MUESTRA MS } \\
\hline & \begin{tabular}{|c|}
$\begin{array}{c}\text { Attu Ret } \\
(\mathrm{m} / \mathrm{s})\end{array}$ \\
\end{tabular} & $\%$ & $\begin{array}{c}\text { Ases Rel } \\
(\mathrm{mm} / \mathrm{s})\end{array}$ & $\%$ & $\begin{array}{c}\text { Aftes Rel } \\
(\mathrm{mn} / \mathrm{s})\end{array}$ & $\%$ & $\begin{array}{c}\text { Arte Re1 } \\
(\mathrm{mm} / \mathrm{s})\end{array}$ & $\%$ \\
\hline Hematita & 0,0821 & 63,54 & 0,0212 & 29,66 & 0,0370 & 33,84 & & \\
\hline Magnet & & & $\begin{array}{l}0,0017 \\
0,0034\end{array}$ & $\begin{array}{l}2,38 \\
476\end{array}$ & & & & \\
\hline Femhid & & & & & 0,0417 & 38,11 & & \\
\hline Goetts & & & & & 0,012 & 11,16 & & \\
\hline mith & 0,0237 & 18,56 & 0,0442 & 6195 & 0.0153 & 10,39 & 0,0167 & 87,30 \\
\hline Monte & 0.0126 & 10,01 & 0,0009 & 1,24 & 0,0032 & 2,89 & 0,0024 & 12,70 \\
\hline \multicolumn{9}{|l|}{ Ilnerita } \\
\hline Museor. & 0,0098 & 7,88 & & & & & & \\
\hline TOTAL & 0,1924 & 100,00 & 0.9714 & 100,00 & 0,1094 & $100 / 0$ & 0.0191 & 100,00 \\
\hline \multicolumn{9}{|c|}{$<2 m m \cdot 50 \mathrm{~cm}$ sin tratar, TA } \\
\hline \multicolumn{3}{|c|}{ MUESTRA M2 } & \multicolumn{2}{|c|}{ MUESTRA M3 } & \multicolumn{2}{|c|}{ MUESTRA M4 } & \multicolumn{2}{|c|}{ MUESTRA MS } \\
\hline & $\begin{array}{l}\text { Area Ret } \\
(\mathrm{mm} / \mathrm{k})\end{array}$ & $\%$ & $\begin{array}{c}\text { Aften Rel } \\
(\mathrm{mm} / \mathrm{s})\end{array}$ & $\%$ & $\begin{array}{c}\text { Asen Rel } \\
(\mathrm{mm} / \mathrm{s})\end{array}$ & \% & $\begin{array}{c}\text { Aret Ret } \\
(\mathrm{mm} / \mathrm{s})\end{array}$ & \% \\
\hline Hemutita & 0,0175 & 65,79 & 0,0244 & 20,10 & 0,0168 & 2491 & & \\
\hline Magretala & & & $\begin{array}{l}0,0018 \\
0,0036\end{array}$ & $\begin{array}{l}2,15 \\
430\end{array}$ & & & & \\
\hline Femhidi & & & & & 0,0226 & 33,38 & & \\
\hline Goetits & & & & & 0,0109 & 16,16 & & \\
\hline mith & 0,0028 & 10,53 & 0,0511 & 61,03 & 0,0142 & 21,01 & 0,0074 & 87,30 \\
\hline Montmot. & 0,0026 & $9,7 n$ & 0,0029 & 3,43 & 0,0031 & 435 & 0,0011 & 12,70 \\
\hline \multicolumn{9}{|l|}{ Ineriats } \\
\hline Muereovit. & 0,037 & 13,91 & & & & & & \\
\hline TOTAL & 0.0266 & 10000 & 0,0838 & $100 \mathrm{~m}$ & 0.0676 & 10000 & oposs & 100.00 \\
\hline
\end{tabular}

La tabla IX lista estimados del porcentaje del contenido total de Fe por peso en las muestras sin tratar a partir de la absorción gamma resonante integrada, suponiendo que todos los minerales poseen el mismo factor-f, fA, y usando estimados para el producto de este factor-f con la fracción de radiación g14,4keV en el haz incidente, G, y de los efectos de saturación. Se utiliza la siguiente relación:

$$
\text { Concentración }(\%)=14.9(\text { Area, } \mathrm{mm} / \mathrm{s})\left(\frac{\text { Area de portamuestra, } \mathrm{cm}^{2}}{\text { masa de la muestra }, \mathrm{mg}}\right)\left(\frac{100}{f_{A} G}\right)
$$




\section{DISCUSIÓN DE RESULTADOS}

La muestra M2 presenta un comportamiento magnético fuerte debido a la presencia de hematita. A TNL no se observa la presencia de óxidos mal cristalizados, lo que significa que este suelo tiene un orden magnético. M3 y M5 presentan hierro paramagnético en mayor proporción. M3 tiene como sextetos los campos magnéticos hiperfinos de la hematita y magnetita.

En la muestra M4 se observa el fenómeno de superparamagnetismo a través de la presencia de la goetita, que es un óxido de Fe mal cristalizado que se encuentra en general como producto de la meteorización de silicatos de Fe+2. En esta misma muestra a TA y sin tratar, la goetita se manifiesta como un doblete paramagnético y a baja temperatura presenta un sexteto magnético.

Es importante notar la variabilidad en la composición mineralógica en las muestras estudiadas a pesar que los sitios donde fueron recolectadas están relativamente cerca.

Merece recalcar la variación de la concentración de Fe en función de la profundidad y tamaño de grano. Por el momento no se cuenta con toda la información necesaria para poder explicar este comportamiento.

\section{CONCLUSIONES}

Este estudio de la composición mineralógica del suelo muestra con claridad la bondad de la espectroscopía Mössbauer para identificar los sesquióxidos de Fe y otros minerales que contienen Fe, y hacer estimados sobre las concentraciones relativas a pesar que éstos se encuentran presentes en bajas concentracio-nes. Los difractogramas proporcionan una primera idea sobre la presencia de minerales mayoritarios pero no hacen posible obtener información detallada y confiable sobre la presencia de los minerales de hierro.

Este trabajo también muestra la necesidad de tomar los espectros Mössbauer a bajas temperaturas para dilucidar la presencia de sesquióxidos de hierro con propiedades superparamagnéticas y confirmar la presencia de otros minerales.

Se muestra el efecto del tratamiento químico por DCB para extraer hierro de minerales con buena cristalización. Estos tratamientos químicos son técnicas auxiliares disponibles que permiten esclarecer la presencia de minerales que contienen hierro cuando estos responden en forma diferenciada a estos tratamientos. Queda pendiente ensayar con otras técnicas químicas disponibles para estos fines.

Asimismo, queda pendiente hacer un análisis más detallado sobre el contenido de arcillas por su importancia para las investigaciones agrícolas. Para este fin será necesario tomar los difractogramas con muestras orientadas a fin de enfatizar la presencia de las arcillas.

Por último, merece estudiar en más detalle la variación de la concentración de Fe en las muestras en función de la profundidad y fracción granulométrica.

\section{RECONOCIMIENTOS}

Las mediciones por EM se llevaron a cabo en el Laboratorio de Espectroscopia Mössbauer de la Facultad de Ciencias Físicas de la Universidad Nacional Mayor de San Marcos y la preparación y tratamientos químicos de las muestras se hicieron en el Laboratorio de Análisis de Suelos de la misma Facultad. Se agradece, también, la colaboración del Centro Brasileiro de Pesquisas Físicas (CBPF) por la toma de los difractogramas de rayos x que han sido utilizados en este estudio. Asimismo, se agradece la colaboración de los miembros del Laboratorio de Suelos de la Universidad Nacional Agraria de La Molina, Lima, Perú, por aportar sus conocimientos para la preparación de las muestras.

\section{BIBLIOGRAFÍA}

1. E. C. Torres Tapia," Aplicaciones de la Espectroscopía Mössbauer en Mineralogía", tesis para optar el título de licenciado en Física, Programa Académico de Física, Universidad Nacional Mayor de San Marcos, (1980)

2. INGEMMET, "Geología de los cuadrángulos de Urubamba y Calca", Boletín № 65 (1996)

3. E. Murad, J. Jhonston ,"Iron Oxides, Mössbauer spectroscopy applied to inorganic chemistry", Vol. 2 (1987)

4. A. Mijovilovich, "Estudio de óxidos de hierro por espectroscopia Mössbauer", tesis de doctorado, Universidad de Buenos Aires, (1997)

5. C. Saragovi, S. Acebal y F. Labenski, "Mossbauer studies on some Argentinian soils: Mollisols from Bahía Blanca", Hyp. Int. 91: 765-769 (1994)

6. R.Vanderberghe, E. De Grave, L.Landuyt, L.Bowen, "Some aspects concerning the characterization of iron oxides and hydroxides in soils and clays" Hyp. Int. 53: 175-196 (1990) 
